\title{
Video Article \\ Rapid Evaluation of Toxicity of Chemical Compounds Using Zebrafish Embryos
}

\author{
Ashok Aspatwar ${ }^{1}$, Milka Marjut Hammaren ${ }^{1}$, Mataleena Parikka ${ }^{1,2}$, Seppo Parkkila ${ }^{1,3}$ \\ ${ }^{1}$ Faculty of Medicine and Health Technology, Tampere University \\ ${ }^{2}$ Oral and Maxillofacial Unit, Tampere University Hospital \\ ${ }^{3}$ Fimlab Ltd, Tampere University Hospital
}

Correspondence to: Ashok Aspatwar at ashok.aspatwar@tuni.fi

URL: https://www.jove.com/video/59315

DOI: doi:10.3791/59315

Keywords: Medicine, Issue 150, Zebrafish embryos, toxicity screening, in vivo toxicity, developmental toxicity, anticancer agents, phenotypic defects, pre-clinical drug development

Date Published: 8/25/2019

Citation: Aspatwar, A., Hammaren, M.M., Parikka, M., Parkkila, S. Rapid Evaluation of Toxicity of Chemical Compounds Using Zebrafish Embryos J. Vis. Exp. (150), e59315, doi:10.3791/59315 (2019).

\section{Abstract}

The zebrafish is a widely used vertebrate model organism for the disease and phenotype-based drug discovery. The zebrafish generates many offspring, has transparent embryos and rapid external development. Zebrafish embryos can, therefore, also be used for the rapid evaluation of toxicity of the drugs that are precious and available in small quantities. In the present article, a method for the efficient screening of the toxicity of chemical compounds using 1-5-day post fertilization embryos is described. The embryos are monitored by stereomicroscope to investigate the phenotypic defects caused by the exposure to different concentrations of compounds. Half-maximal lethal concentrations $\left(\mathrm{LC}_{50}\right)$ of the compounds are also determined. The present study required 3-6 $\mathrm{mg}$ of an inhibitor compound, and the whole experiment takes about 8-10 $\mathrm{h}$ to be completed by an individual in a laboratory having basic facilities. The current protocol is suitable for testing any compound to identify intolerable toxic or off-target effects of the compound in the early phase of drug discovery and to detect subtle toxic effects that may be missed in the cell culture or other animal models. The method reduces procedural delays and costs of drug development.

\section{Video Link}

The video component of this article can be found at https://www.jove.com/video/59315/

\section{Introduction}

Drug development is an expensive process. Before a single chemical compound is approved by the Food and Drug Administration (FDA) and European Medicines Agency (EMA) several thousand compounds are screened at a cost of over one billion dollars ${ }^{1}$. During the preclinical development, the largest part of this cost is required for the animal testing ${ }^{2}$. To limit the costs, researchers in the field of drug development need alternative models for the safety screening of chemical compounds ${ }^{3}$. Therefore, in the early phase of the drug development, it would be very beneficial to use a method that can rapidly evaluate the safety and toxicity of the compounds in a suitable model. There are several protocols that have been used for the toxicity screening of chemical compounds involving animal and cell culture models but there is not a single protocol that is validated and is in common use ${ }^{4,5}$. Existing protocols using zebrafish vary in length and have been used by individual researchers who evaluated the toxicity as per their convenience requirement ${ }^{6,7,8,9,10,11,12}$.

In the recent past, the zebrafish has emerged as a convenient model for the evaluation of the toxicity of chemical compounds during embryonic development ${ }^{6,7}$. The zebrafish has many in-built advantages for the evaluation of chemical compounds ${ }^{13}$. Even large-scale experiments are amenable, as a zebrafish female can lay batches of 200-300 eggs, which develop rapidly ex vivo, do not need external feeding for up to a week and are transparent. The compounds can be added directly into the water, where they can (depending on the nature of the compound) diffuse through the chorion, and after hatching, through the skin, gills and mouth of larvae. The experiments do not require copious amounts of chemical compounds ${ }^{14}$ due to the small size of the embryo. Developing zebrafish embryos express most of the proteins required to achieve the normal developmental outcome. Therefore, a zebrafish embryo is a sensitive model to assess whether a potential drug can disturb the function of a protein or signaling molecule that is developmentally significant. The organs of the zebrafish become functional between $2-5 \mathrm{dpf}{ }^{15}$, and compounds that are toxic during this sensitive period of embryonic development induce phenotypic defects in zebrafish larvae. These phenotypic changes can be readily detected using a simple microscope without invasive techniques ${ }^{11}$. Zebrafish embryos are widely used in toxicological research due to their much greater biological complexity compared to in vitro drug screening using cell culture models ${ }^{16,17}$. As a vertebrate, the genetic and physiologic makeup of zebrafish is comparable to humans and hence toxicities of chemical compounds are similar between zebrafish and humans $8,18,19,20,21,22$. Zebrafish is, thus, a valuable tool in the early phase of drug discovery for the evaluation of toxicity and safety of the chemical compounds.

In the present article, we provide a detailed description of the method used for evaluating the safety and toxicity of carbonic anhydrase (CA) inhibitor compounds using 1-5-day post fertilization (dpf) zebrafish embryos by a single researcher. The protocol involves exposing zebrafish embryos to different concentrations of chemical inhibitor compounds and studying the mortality and phenotypic changes during the embryonic 
development. At the end of the exposure to the chemical compounds, the $\mathrm{LC}_{50}$ dose of the chemical is determined. The method allows an individual to carry out efficient screening of 1-5 test compounds and takes about 8-10 h depending on the experience of the person with the method (Figure 1). Each of the steps required to assess the toxicity of the compounds is outlined in Figure 2. The evaluation of toxicity of CA inhibitors requires 8 days, and includes setting up of mating pairs (day 1); collection of embryos from breeding tanks, cleaning and transferring them to $28.5^{\circ} \mathrm{C}$ incubator (day 2); distribution of the embryos into the wells of a 24-well plate and addition of diluted CA inhibitor compounds (day 3 ); phenotypic analysis and imaging of larvae (day 4-8), and determination of $\mathrm{LC}_{50}$ dose (day8). This method is rapid and efficient, requires a small amount of the chemical compound and only basic facilities of the laboratory.

\section{Protocol}

The zebrafish core facility at Tampere University has an establishment authorization granted by the National Animal Experiment Board (ESAVI/7975/04.10.05/2016). All the experiments using zebrafish embryos were performed according to the Provincial Government of Eastern Finland, Social and Health Department of Tampere Regional Service Unit protocol \# LSLH-2007-7254/Ym-23.

\section{Setting Up of Overnight Zebrafish Mating Tanks}

1. Place 2-5 adult male zebrafish and 3-5 adult female zebrafish into mating tanks overnight. (Breeding is induced in the morning by automatic dark and light cycle overnight).

2. Set up several crosses to obtain enough embryos for assessing the toxicity of more than two chemical compounds. For the evaluation of toxicity, each concentration needs a minimum of 20 embryos $^{23}$.

3. To avoid handling stress to the animals, allow the animals to rest for 2 weeks before using the same individuals for breeding.

\section{Collection of Embryos and Preparing Plates for Exposure to the Chemical Compounds}

1. Collect the embryos, the next day before noon, using a fine-mesh strainer and transfer them onto a Petri dish containing E3 embryo medium [5.0 mM NaCl, $0.17 \mathrm{mM} \mathrm{KCl}, 0.33 \mathrm{mM} \mathrm{CaCl}_{2}, 0.33 \mathrm{mM} \mathrm{MgSO}_{4}$, and $0.1 \%$ w/v Methylene Blue].

2. Remove debris using a plastic Pasteur pipette (e.g., food and solid waste). Examine each batch of embryos under the stereomicroscope to remove the unfertilized/dead embryos (identified by their opaque appearance).

3. Keep the embryos at $28.5^{\circ} \mathrm{C}$ in an incubator. Examine the embryos, the next morning, under a stereomicroscope and remove any unhealthy or dead embryos. Also, replace the old E3 medium with fresh E3 medium.

NOTE: Zebrafish embryos are always maintained at $28.5^{\circ} \mathrm{C}$ under laboratory conditions.

4. Carefully transfer 1 embryo into each well of a 24-well plate containing enough E3 medium to cover the embryos.

\section{Preparations of the Stock Solution of Chemical Compounds and Distribution of Diluted Compound into the Wells}

1. Take out the vials containing inhibitor compounds stored at $4{ }^{\circ} \mathrm{C}$.

NOTE: Depending on the properties of the compound, these are stored at different temperatures.

2. Weigh the compound(s) using an analytical balance that can weigh a few milligrams ( $\mathrm{mg}$ ) of the compound accurately.

3. Prepare at least $250 \mu \mathrm{L}(100 \mathrm{mM})$ of stock solution for each compound in an appropriate solvent (e.g., E3 water or Dimethyl sulfoxide (DMSO), based on the solubility properties of the compounds.

NOTE: The above steps can be done a day before the start of the experiment at a convenient time and stored at $4{ }^{\circ} \mathrm{C}$ ).

4. Make serial dilutions of the stock solutions (e. g., $10 \mu \mathrm{M}, 20 \mu \mathrm{M}, 50 \mu \mathrm{M}, 100 \mu \mathrm{M}, 150 \mu \mathrm{M}, 300 \mu \mathrm{M}$ and $500 \mu \mathrm{M}$ ) using $\mathrm{E} 3$ water in $15 \mathrm{~mL}$ centrifuge tubes.

NOTE: The concentrations and number of serial dilutions vary from one compound to another compound depending on their toxicity levels.

5. From the 24 well plate containing embryos, remove E3 water from the wells using a Pasteur pipette and a $1 \mathrm{~mL}$ pipette (containing $1 \mathrm{dpf}$ embryos) one row at a time.

6. Distribute $1 \mathrm{~mL}$ of each diluent in each well (starting from lower and moving to higher concentration) into the wells of 24-well plate.

7. Set up a control group from the same batch of embryos and add the corresponding amount of diluent.

8. Label 24-well plates with the name and concentration of the compound and keep the plates at $28.5^{\circ} \mathrm{C}$ in an incubator.

\section{Phenotypic Analysis and Imaging of the Embryos Using a Stereomicroscope}

1. Examine the embryos under a stereomicroscope for parameters $24 \mathrm{~h}$ after exposure to the chemical compounds.

1. Note the parameters such as mortality, hatching, heartbeat, utilization of yolk sack, swim bladder development, movement of the fish, pericardial edema, and shape of the body ${ }^{23}$.

2. Take the larvae exposed to each concentration of the compound and lay them sideways in a small Petri dish containing $3 \%$ high molecular weight methyl cellulose using a metal probe.

NOTE: The $3 \%$ methyl cellulose (high molecular with) is a viscous liquid needed for embedding the fish with a required orientation for microscopic examination. For orienting the fish in this liquid, a metal probe is needed.

3. Take the images using stereomicroscope attached to a camera. Save the images in a separate folder each day till the end of the experiment.

4. Enter all the observations in a table each day either in an online table or on a printed sheet.

5. If the compounds are neurotoxic, the 4 to $5 \mathrm{dpf}$ larvae may show altered swim pattern, make a record of such changes either by capturing a short ( $30 \mathrm{~s}$ to $1 \mathrm{~min}$ ) video of the larvae exhibiting abnormal movement pattern. 
6. After 5 days of exposure to the chemical compounds, note the concentration at which half of the embryos die for calculating the half maximal lethal concentration $50\left(\mathrm{LC}_{50}\right)$ of each chemical.

NOTE: The $\mathrm{LC}_{50}$ is the concentration at which $50 \%$ of the embryos die at the end of 5 days of exposure to a chemical compound. Use a minimum of 20 embryos for testing the toxicity of each concentration of a compound ${ }^{23}$.

7. Construct a curve for mortality of embryos for all the concentrations using a suitable program.

\section{Representative Results}

The critical part of the evaluation of toxicity is testing different concentrations of one or multiple chemical compounds in a single experiment. In the beginning, select the compounds for evaluation of toxicity, the number of concentrations to test for each compound, and accordingly, make a chart (Figure 3). We used a unique color for each compound to organize the samples (Figure 3). The use of solvent resistant marker and labeling at the bottom or sides of the plates is important to avoid mix-up later. If the compounds induce any phenotypic defects in the larvae exposed to different concentrations of inhibitors, the defects are recorded every $24 \mathrm{~h}$ over the period of 1-5 dpf (Figure 4A,B,C,D). The embryos treated with a known CA IX inhibitor at a concentration of $500 \mu \mathrm{M}$ did not show any apparent phenotypic changes in the 1-5 days of exposure to the chemical compound (Figure 4B). Figure 4C and Figure 4D shows the embryos treated with $\beta$-CA inhibitors that induced various phenotypic defects unhatched embryos even at day 3 (arrow head), curved body structure (arrow), unutilized yolk sack and pericardial edema (arrow head) and absence of otolith sacs in the larvae at 5 days after treatment with chemical compound (arrow). In another study, the embryos treated with CA inhibitor (Figure 4E) shows the absence of otolith sacs and swim bladder (arrow heads). In our study, (Figure 4C,D), we documented phenotypic defects (fragile embryos and absence of pigmentation) even after day 1 of exposure to CA inhibitors. The phenotypic analyses showed that some of the inhibitor compounds are lethal and cannot be developed as drugs for human use (Figure 4C,D and Table 1).

The experiments identified a representative compound which induced minimal or no phenotypic changes during embryonic development and showed a high $\mathrm{LC}_{50}$ dose (Figure 5), suggesting that the compound is safe for further characterization and can be potentially developed into a drug candidate for human use ${ }^{16}$. Looking at still images of larvae exposed to the compound showed no phenotypic defects (Figure 4B). However, the same compound was found to be neurotoxic and induced ataxia in the larvae after 5-days of exposure to the CA inhibitor (Figure 6). This phenotype could only be detected by directly observing the swimming behavior of the zebrafish larvae under the microscope. These studies suggested that the neural development of zebrafish larvae is sensitive to the compound ${ }^{16,17}$.

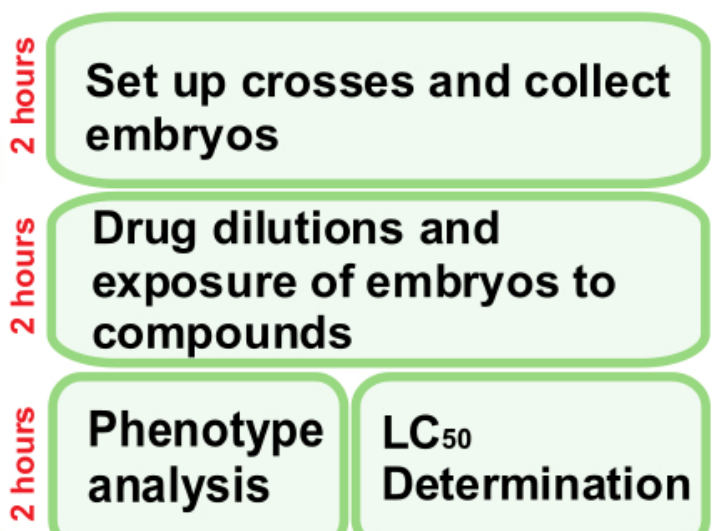

Figure 1: Time in hours required for the rapid screening of chemical compounds using zebrafish embryos: In one set of experiments, a person with hands-on experience can screen about 5 chemical compounds (each compound requiring a minimum of 6 dilutions) using zebrafish embryos in 24-well plates. In total, it takes about $8-10 \mathrm{~h}$ from the setting up of crosses to performing $\mathrm{LC}_{50}$ determination over a period of 8 days.

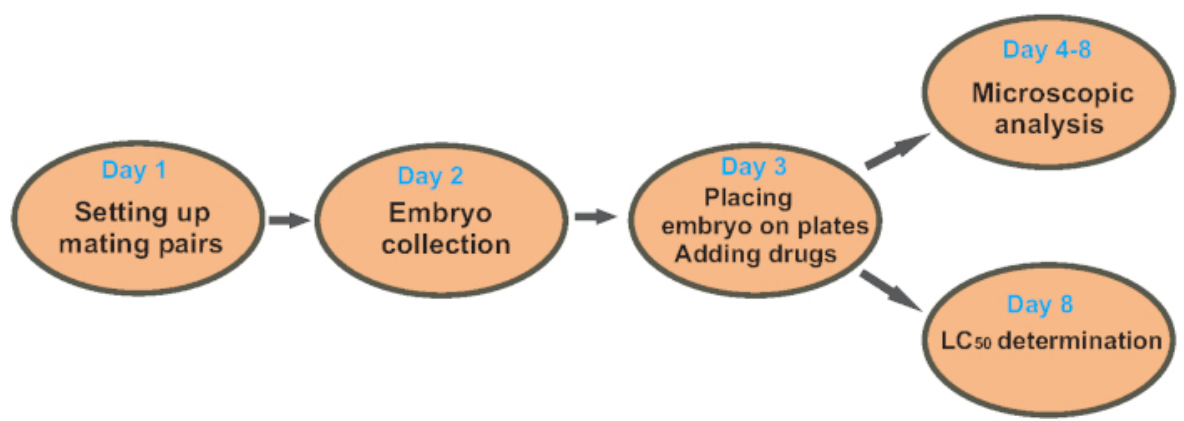

Figure 2: Chart showing the breakdown of toxicity evaluation of chemical compounds. Toxicity screening of chemical compounds requires 8 days and can be broken down into five steps. (Day 1) Consists of setting up of zebrafish mating pairs in tanks. (Day 2) Involves collecting embryos from the mating tanks into Petri dishes followed by keeping them at a $28.5^{\circ} \mathrm{C}$ incubator for overnight. (Day 3 ) Examination of embryos using a stereomicroscope and cleaning the embryos. Distribution of the embryos into 24 -well plates and adding the dilutions of the test

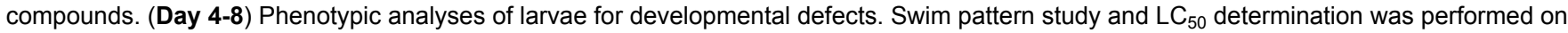
the last day of exposure to compounds. Please click here to view a larger version of this figure. 


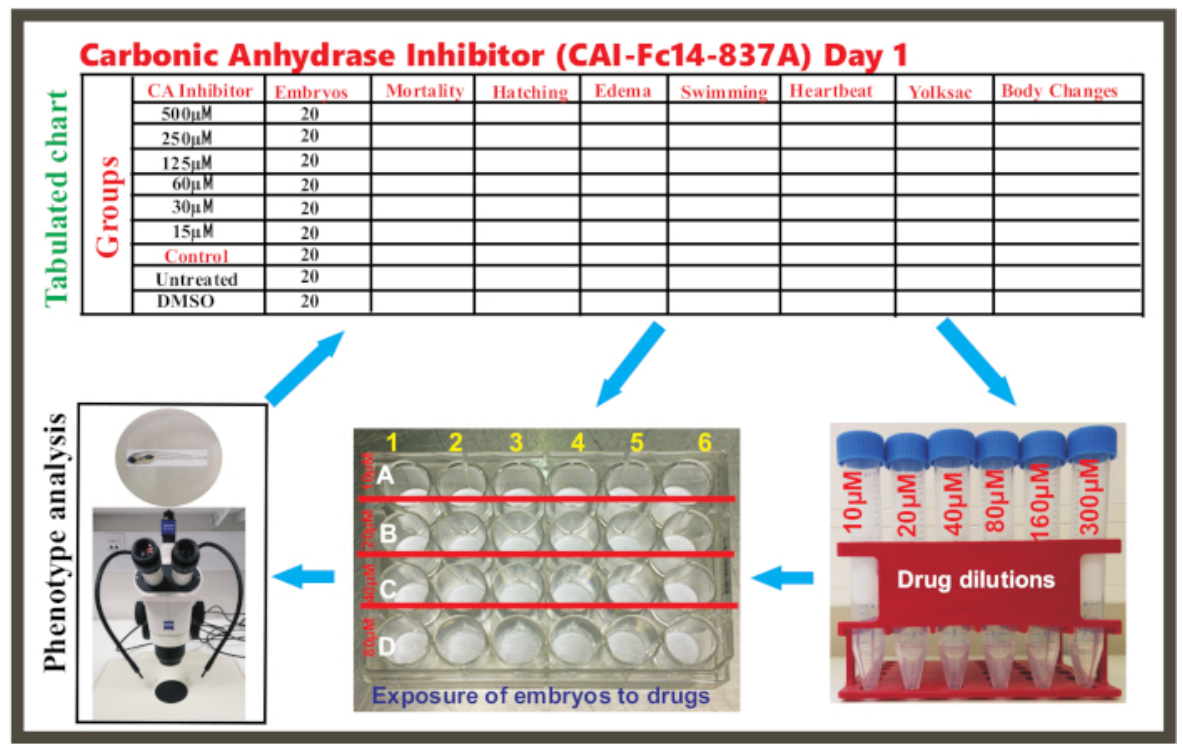

Figure 3: Schematic presentation of experiments involved in toxicity evaluation. The toxicity evaluation consists of preparing tabulated chart containing information about chemical compounds, dilutions of the compounds and parameters to be assessed. Making the dilution of stock solutions to the desired concentrations in $15 \mathrm{~mL}$ centrifuge tubes (the dilution from one tube to be added to each row of the 24-well plate). A 24well plate marked with a water proof marker with the name of the test compound, concentration in each row, and date of exposure. Examination and imaging of embryos by transferring the larvae onto a small Petri dish containing $3 \%$ high molecular weight methyl cellulose. Please click here to view a larger version of this figure.
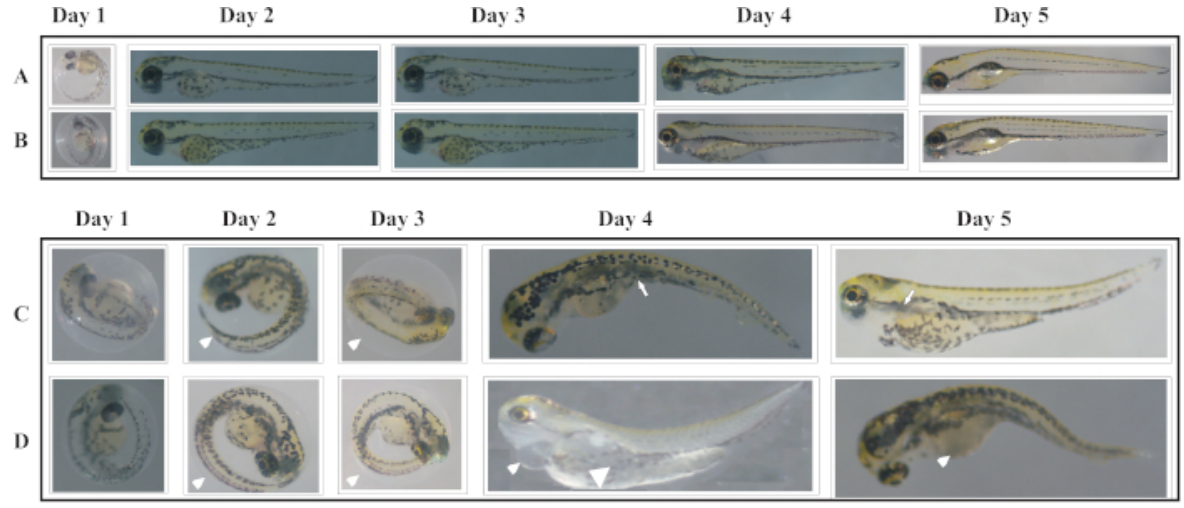

Day 5

Day 5

E

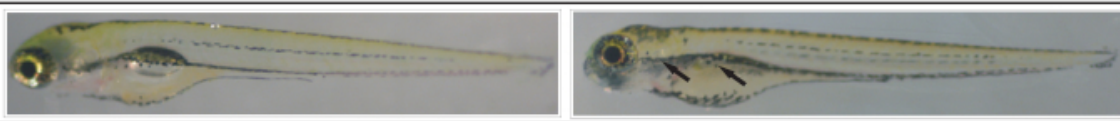

Figure 4: Example of phenotypic analysis of the larvae in the control and test compound treated groups. (A) Phenotypic analyses of the control group larvae not treated with any compound showed no phenotypic defect under a microscope. (B) Larvae treated with $500 \mu \mathrm{M} C A$ inhibitor (known inhibitor of carbonic anhydrase IX showed no observable phenotypic defects. (C, D) The developing larvae treated with $\beta$-CA inhibitor with concentrations of $250 \mu \mathrm{M}$ and $125 \mu \mathrm{M}$ respectively. The compounds induced phenotypic defects such as curved body, pericardial edema, and unutilized yolk sac (arrows and arrow heads). Panels $\mathbf{A}$ and $\mathbf{B}$ has been modified from Aspatwar et al ${ }^{16}$. The panels $\mathbf{C}, \mathbf{D}$ and $\mathbf{E}$ show previously unpublished images, which were obtained using a different microscope. Please click here to view a larger version of this figure. 


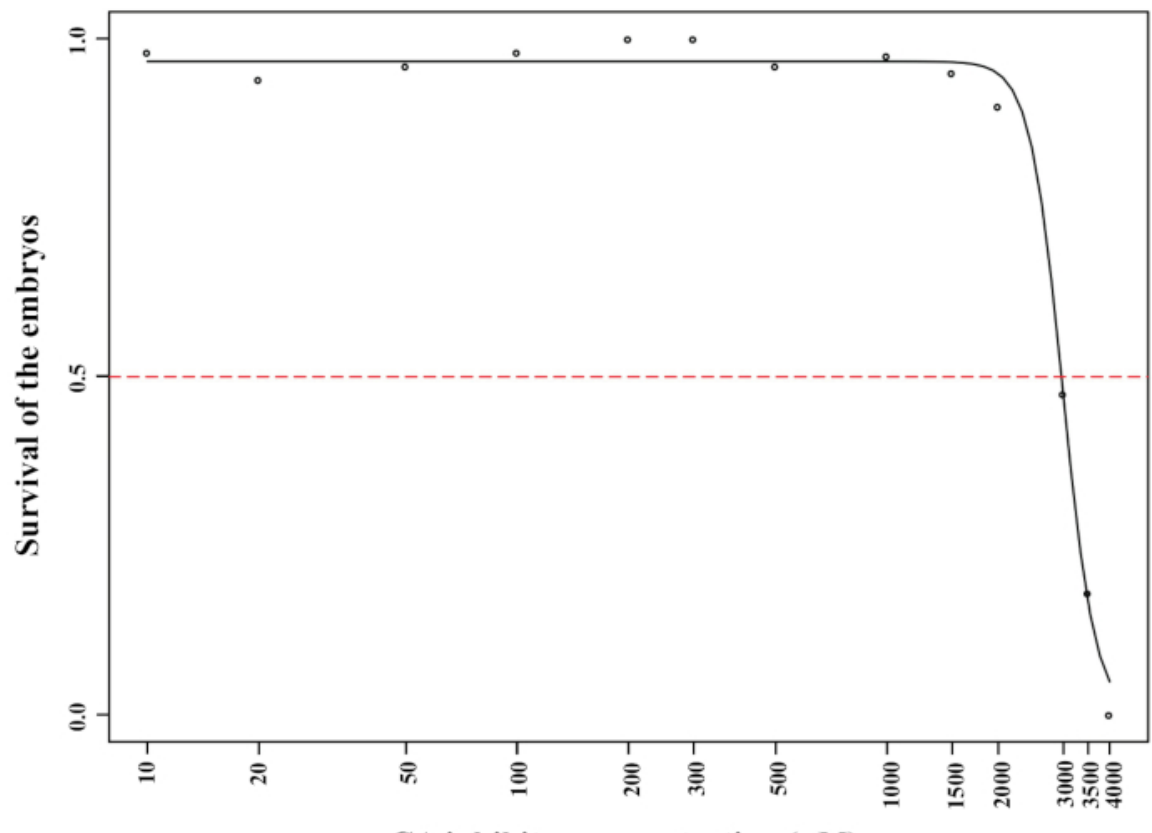

CA inhibitor concentration $(\mu \mathrm{M})$

Figure 5: Determination of lethal concentration $50\left(\mathrm{LC}_{50}\right)$ using 1-5 dpf zebrafish larvae. Toxicity assessment using zebrafish embryos allows researchers to determine the minimum lethal concentration of the chemical compound at the end of the experiment. The $\mathrm{LC}_{50}$ concentration of the compound (a known CA IX inhibitor) was $3.5 \mathrm{mM}$. The high $\mathrm{LC}_{50}$ concentration allows further characterization of the compound. This figure has been modified from Aspatwar et al. ${ }^{16}$. Please click here to view a larger version of this figure.

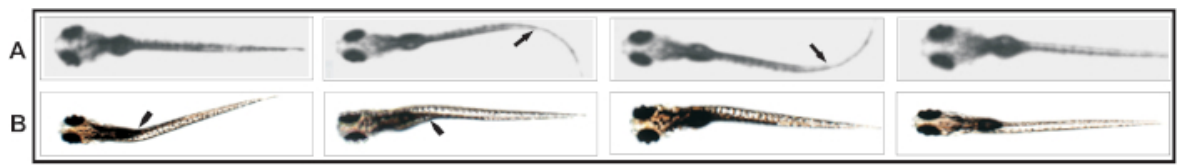

Figure 6: An example of swim pattern analysis in both untreated (A) and inhibitor treated (B) groups of larvae. The zebrafish larvae treated in panel B with $300 \mu \mathrm{M}$ test inhibitor compound (a known inhibitor of human carbonic anhydrase IX) showed an abnormal (ataxic) movement pattern (arrow heads), suggesting that the compound induces neurotoxicity in the developing embryos. The arrowheads point to the normal curvature of the tail during swimming. In panel A the arrows point to the normal curvature of the tail during swimming. This figure has been modified from Aspatwar et al. ${ }^{16}$. Please click here to view a larger version of this figure.

\begin{tabular}{|c|c|c|c|c|c|}
\hline CA inhibitor & Toxicity Screening & $\mathrm{LC}_{50}$ & In vivo & Toxic CAls & Reference \\
\hline Carbamates & 24 & All & $1^{a}$ & $3^{b}$ & ${ }^{24}$, Unpublished \\
\hline Coumarins & 10 & All & - & 2 & Unpublished \\
\hline Nitroimidazoles & 2 & All & 2 & $2^{c}$ & 16 \\
\hline Sulfonamides & 5 & All & - & 3 & 25 \\
\hline Total & 41 & All & 3 & 10 & - \\
\hline
\end{tabular}

Table 1: A summary of the safety and toxicity of the compounds screened. The toxicity evaluation experiment helps the researcher to reach a conclusion about the safety of the tested chemical compounds. The $\mathrm{LC}_{50}$ concentration allows to define safe concentration for further characterization. A researcher can decide if the compound is lethal even after $24 \mathrm{~h}$ of exposure of embryos to the compound under investigation. In our example, a subtle effect on swimming due to neurotoxicity is the significant information, which is helpful for setting up further experiments. Accordingly, based on the toxicity screening, we were able to use safe concentrations of the CA inhibitor for further characterization in vivo ${ }^{24}$.

\section{Discussion}

In vitro toxicity test using cultured cells can detect survival and morphological studies of the cells providing limited information about the toxicity induced by the test compound. The advantage of toxicity screening of chemical compounds using zebrafish embryos is rapid detection of chemically induced phenotypic changes in a whole animal during embryonic development in a relevant model organism. Approximately $70 \%$ of protein-coding human genes have orthologs counterparts in the zebrafish genome ${ }^{25}$. Genetic pathways controlling the signal transduction and 
development are highly conserved between human and zebrafish ${ }^{26}$, and, therefore, chemical compounds are likely to have similar toxic effects in humans ${ }^{25}$.

Zebrafish are at the forefront of toxicological research and have already been extensively used to detect toxins in water samples and to study the mechanism of action of environmental toxins and their effects on the animal ${ }^{27}$. In this article, we show the use of developing zebrafish embryos to evaluate the toxicity of potential compounds at the early phase of drug discovery. Our rapid and multifaceted toxicity assessment is based on 1) changes in the phenotype of the organism (hatching, otolith sacs, pericardial edema, yolk sac utilization, notochord development, heartbeat, swim bladder development, movement) during embryonic development over a period of 5 days, and 2) determination of the lethal concentration $\left(\mathrm{LC}_{50}\right)$. Reasonable hours of hands-on work (8-10 h divided over 8 days) is enough to determine the toxicity profile of a compound using this procedure. For newly synthesized compounds that are available in limited amounts, toxicity can be evaluated using a minimum of 3 $\mathrm{mg}$ of the compound. No special equipment is required. The procedure is, thus, a rapid and low-cost way to evaluate the toxic effects of any compound that can potentially be developed into the drug for human use.

The quality of the batch of embryos has a great impact on the outcome of the experiment. The quality of eggs (including the rate of fertilization) is not obvious immediately after collection from the breeding tanks (0-4 hpf). For obtaining only normally developing embryos for the experiments, before adding compounds, we allow the embryos to remain at $28.5^{\circ} \mathrm{C}$ for $24 \mathrm{~h}$ after the collection from the breeding tanks. After $24 \mathrm{hpf}$, any dead or unhealthy-looking embryos are discarded. In addition to this, it is advisable to have a mock-treated group of embryos in each experiment to further control for the quality of the batch of eggs used in the experiment as well as to see the baseline mortality to accurately determine $\mathrm{LC}_{50}$.

A mock-treated group is also needed to control for the toxicity of the vehicle of choice. Many chemicals are insoluble in water and DMSO is often used as the vehicle for the delivery of the test compounds. DMSO is generally well tolerated by the embryos in lower $(0.1 \%)$ concentrations ${ }^{28}$. Sometimes, the compounds are not completely soluble even in DMSO and the solution appears cloudy making it difficult for the evaluation of the toxicity. In such cases, to get the stock solution of the compound completely soluble and clear, adding a drop of $0.1 \% \mathrm{NaOH}$ will solve the problem. Appropriate control groups need to be set up for assessing the toxicity of the compound accurately. If other vehicles are used, their inherent toxicity might affect the experiment.

Each well of a 24-well plate contains from 1-10 embryos submerged in a total volume of $1 \mathrm{~mL}$. If the test compound is available only in small amounts, it may be necessary to use up to 10 embryos per well for evaluating its toxicity. It is highly recommended that only 1 embryo per well should be used for each analysis ${ }^{16,24,29}$. The plate is stored at $28.5^{\circ} \mathrm{C}$ incubator for 5 days. Sometimes significant evaporation is observed causing a marked change in the concentration of the compound in a given well ${ }^{16}$. By sealing the 24 -well plates with paraffin films from all sides, placing embryos only in the middle wells and filling the other wells along the rims with water, the problems caused by evaporation can be avoided. In our earlier studies, we did not observe any evaporation of the diluents of the chemicals from 24 -well plates ${ }^{24,29}$. Also, if the compound in question is known to be unstable under ambient temperatures, daily changes of water with fresh compound will be needed for reliable results.

The swim pattern of zebrafish larvae is analyzed after the 5 days of exposure to the compound. The wells of a 24-well plate containing 5 dpf larvae are not ideal for the purpose due to the limited size of the well. Therefore, for accurate analysis of swim pattern, the larvae need to be transferred to a Petri dish containing $50 \mathrm{~mL}$ of E3 water and can settle for 2 min before the analysis. In our study, only two inhibitors showed the effect on swim pattern ${ }^{16}$ among the $52 \alpha-C A$ and $\beta-C A$ inhibitors screened for safety and toxicity, based on our experience this test can detect subtle changes including mild ataxic movement of the larvae.

This study demonstrated that the only limitation to the current method is physical dexterity. This concern can be overcome through repetition, as the skill of a person improves with practice. Once the expertise is achieved the evaluation of compounds using zebrafish embryos is ethical, easy, efficient and informative. We, therefore, expect that such a rapid assay using zebrafish embryos will become a popular tool for the in vivo toxicity screening in the early phase of drug development.

\section{Disclosures}

No potential conflict of interest was reported by the authors.

\section{Acknowledgments}

The work was supported by grants from Sigrid Juselius Foundation (SP, MP), Finnish Cultural Foundation (AA, MH), Academy of Finland (SP, MP), Orion Farmos Foundation (MH), Tampere Tuberculosis Foundation (SP, MH and MP) and Jane and Aatos Erkko Foundation (SP and MP). We thank our Italian and French collaborators, Prof. Supuran, and Prof. Winum, for providing carbonic anhydrase inhibitors for safety and toxicity evaluation for anti-TB and anti-cancer drug development purposes. We thank Aulikki Lehmus and Marianne Kuuslahti for the technical assistance. We also thank Leena Mäkinen and Hannaleena Piippo for their help with the zebrafish breeding and collection of embryos. We sincerely thank Harlan Barker for critical evaluation of the manuscript and insightful comments.

1. Amaouche, N., Casaert Salome, H., Collignon, O., Santos, M. R., Ziogas, C. Marketing authorization applications submitted to the European Medicines Agency by small and medium-sized enterprises: an analysis of major objections and their impact on outcomes. Drug Discovery Today. 23 (10), 1801-1805 (2018).

2. Garg, R.C., Bracken, W.M., Hoberman, A.M. Reproductive and developmental safety evaluation of new pharmaceutical compounds. In: Reproductive and Developmental Toxicology. Gupta RC, Ed. Elsevier: Boston, MA, USA, 89-109 (2011).

3. Lee, H. Y., Inselman, A. L., Kanungo, J., Hansen, D. K. Alternative models in developmental toxicology. Systems Biology in Reproductive Medicine. 58 (1), 10-22 (2012). 
4. Gao, G., Chen, L., Huang, C., Anti-cancer drug discovery: update and comparisons in yeast, Drosophila, and zebrafish. Current Molecular Pharmacology. 7 (1), 44-51 (2014).

5. Brown, N. A. Selection of test chemicals for the ECVAM international validation study on in vitro embryotoxicity tests. European Centre for the Validation of Alternative Methods. Alternatives to Laboratory Animals. 30 (2), 177-98 (2002).

6. Selderslaghs, I. W., Van Rompay, A. R., De Coen, W., Witters, H. E. Development of a screening assay to identify teratogenic and embryotoxic chemicals using the zebrafish embryo. Reproductive Toxicology. (Elmsford, N.Y.) 28 (3), 308-320 (2009).

7. Brannen, K. C., Panzica-Kelly, J. M., Danberry, T. L., Augustine-Rauch, K. A. Development of a zebrafish embryo teratogenicity assay and quantitative prediction model. Birth Defects Research Part B Developmental and Reproductive Toxicology. 89 (1), 66-77 (2010).

8. Hermsen, S. A., van den Brandhof, E. J., van der Ven, L. T., Piersma, A. H. Relative embryotoxicity of two classes of chemicals in a modified zebrafish embryotoxicity test and comparison with their in vivo potencies. Toxicology in Vitro. 25 (3), 745-753 (2011).

9. Lessman, C. A. The developing zebrafish (Danio rerio): a vertebrate model for high-throughput screening of chemical libraries. Birth Defects Research. Part C, Embryo Today: Reviews. 93 (3), 268-80 (2011).

10. Lantz-McPeak, S., et al. Developmental toxicity assay using high content screening of zebrafish embryos. Journal of Applied Toxicology. 35 (3), 261-72 (2015)

11. Truong, L., Harper, S. L., Tanguay, R. L., Evaluation of embryotoxicity using the zebrafish model. Methods in Molecular Biology. 691, 271-279 (2011).

12. Rodrigues, G. C. et al. Design, synthesis, and evaluation of hydroxamic acid derivatives as promising agents for the management of Chagas disease. Journal of Medicinal Chemistry. 57 (2), 298-308 (2014).

13. Kanungo, J., Cuevas, E., Ali, S. F., Paule, M. G. Zebrafish model in drug safety assessment. Current Pharmaceutical Design. 20 (34), 5416-5429 (2014).

14. Peterson, R. T., Link, B. A., Dowling, J. E., Schreiber, S. L. Small molecule developmental screens reveal the logic and timing of vertebrate development. Proceedings of the National Academy of Sciences of the United States of America. 97 (24), 12965-12969 (2000).

15. Stainier, D. Y., Fishman, M. C. The zebrafish as a model system to study cardiovascular development. Trends in Cardiovascular Medicine. 4 (5), 207-212 (1994).

16. Aspatwar, A. et al. Nitroimidazole-based inhibitors DTP338 and DTP348 are safe for zebrafish embryos and efficiently inhibit the activity of human CA IX in Xenopus oocytes. Journal of Enzyme Inhibition and Medicinal Chemistry. 33 (1), 1064-1073 (2018).

17. Rami, M. et al. Hypoxia-targeting carbonic anhydrase IX inhibitors by a new series of nitroimidazole-sulfonamides/sulfamides/sulfamates. Journal of Medicinal Chemistry. 56 (21), 8512-8520 (2013).

18. Spitsbergen, J. M., Kent, M. L. The state of the art of the zebrafish model for toxicology and toxicologic pathology research--advantages and current limitations. Toxicologic Pathology. 31 Suppl, 62-87 (2003).

19. Teraoka, H. et al. Induction of cytochrome P450 1A is required for circulation failure and edema by 2,3,7,8-tetrachlorodibenzo-p-dioxin in zebrafish. Biochemical and Biophysical Research Communications. 304 (2), 223-228 (2003).

20. Zon, L. I., Peterson, R. T. In vivo drug discovery in the zebrafish. Nature Reviews Drug Discovery. 4 (1), 35-44 (2005).

21. Hill, A. J., Teraoka, H., Heideman, W., Peterson, R. E. Zebrafish as a model vertebrate for investigating chemical toxicity. Toxicological Sciences. 86 (1), 6-19 (2005).

22. Kari, G., Rodeck, U., Dicker, A. P. Zebrafish: an emerging model system for human disease and drug discovery. Clinical Pharmacology and Therapeutics. 82 (1), 70-80 (2007).

23. Gourmelon, A., Delrue, N. Validation in Support of Internationally Harmonised OECD Test Guidelines for Assessing the Safety of Chemicals Advances in Experimental Medicine and Biology. 856, 9-32 (2016).

24. Aspatwar, A. et al. beta-CA-specific inhibitor dithiocarbamate Fc14-584B: a novel antimycobacterial agent with potential to treat drug-resistant tuberculosis. Journal of Enzyme Inhibition and Medicinal Chemistry. 32 (1), 832-840 (2017).

25. Kazokaite, J., Aspatwar, A., Kairys, V., Parkkila, S., Matulis, D., Fluorinated benzenesulfonamide anticancer inhibitors of carbonic anhydrase IX exhibit lower toxic effects on zebrafish embryonic development than ethoxzolamide. Drug and Chemical Toxicology. 40 (3), $309-319$ (2017).

26. Howe, K. et al. The zebrafish reference genome sequence and its relationship to the human genome. Nature. 496 (7446), $498-503$ (2013).

27. Granato, M., Nusslein-Volhard, C. Fishing for genes controlling development. Current Opinion in Genetics \& Development. 6 (4), $461-468$ (1996).

28. Bambino, K., Chu, J. Zebrafish in Toxicology and Environmental Health. Current Topics in Developmental Biology. 124, 331-367 (2017).

29. Goldsmith, P. Zebrafish as a pharmacological tool: the how, why and when. Current Opinion in Pharmacology. 4 (5), $504-512$ (2004). 